\title{
Relationship between metaplastic changes and occurrence of endocrin cells, lysozyme and lactoferrin in gallbladder carcinoma
}

\section{ORIGINAL ARTICLE Annals of Cancer Research and Therapy}

\author{
Katsutaka Sai - Masakuni Onda - Yoshiyuki Ozawa - Tatsuya Honjo · \\ Tadashi Okuda $\cdot$ Yoshinobu Sumiyama*
}

\begin{abstract}
The association between metaplastic changes seen in gallbladder cancer on the one hand and the appearance of endocrine cells, lysozyme and lactoferrin on the other was studied using serial sections of 50 cases of gallbladder cancer $(9 / 50$, early stage). The sections were examined histologically and immunohistochemically for the purpose of elucidating the histogenesis of gallbladder cancer. Gastrin-secreting, somatostatin-secreting and serotonin-secreting cells in cancer lesions were observed in each of 14 cases $(28 \%), 10$ cases $(20 \%)$ and 15 cases $(30 \%)$ and those in the non-cancerous mucosa each in 19 cases $(38 \%)$, 16 cases $(32 \%)$ and 19 cases $(38 \%)$. Motilin-secreting cells were not observed in cancer lesions and were observed in only 2 cases $(4 \%)$ in non-cancerous mucosa. The immunohistochemical activity of lysozyme and lactoferrin were observed in 29 cases $(58 \%)$ and 6 cases $(12 \%)$, respectively, in the cancer lesions and in 29 cases $(58 \%)$ and 4 cases $(8 \%)$, respectively, in the non-cancerous mucosa. No difference in the appearance rate of metaplastic cells was noticed between advanced and early gallbladder cancer. Metaplastic changes of endocrine cells and lysozyme are closely related to the development of gallbladder cancer. It is therefore possible that the intestinal metaplasia of gallbladder mucosa is an important lesion as regards carcinogenesis.
\end{abstract}

Ann Cancer Res Ther 7(1) : 34 38, 1988/Received 25 May 1998, Accepted 5 Jun 1998 Key words : gallbladder cancer, metaplastic change, endocrin cell, lysozyme, lactoferrin

Regarding the histogenesis of gallbladder cancer, metaplastic changes, particularly intestinal metaplasia have been considered as important background lesions ${ }^{1,2)}$. To discuss carcinogenesis not only in the gallbladder but also in the intestinal tract, it is necessary to observe the behavior of the mucosal epithelium itself and to understand the diversity of cell differentiation. Therefore, the present study is a histopathological study of resected specimens of gallbladder cancer. The cancerous and noncancerous parts of these specimens were examined histopathologically and immunohistochemically in order to clarify the relationship between gallbladder cancer and metaplastic changes.

\section{Materials and methods}

9 cases of early gallbladder cancer, in which infiltration does not go beyond the tunica muscularis propia, and 41 cases of advanced cancer, in which infiltration goes beyond the tunica muscularis propia but remains in the gallbladder wall, were studied. 16 males and 34 females

\footnotetext{
* The 3rd Department of Surgery, School of Medicine, Toho University Hospital at Ohashi

Correspondence to : Katsutaka Sai, The 3rd Department of Surgery, School of Medicine. Toho University Hospital at Ohashi. 2-17-6 Ohashi Meguro-ku Tokyo Japan
}

participated in the study; the range of age was from 39 to 84 years (mean age of 66.8 years). All resected gallbladders were immediately incised on the surface of the liver bed from the neck to the fundus. After washing, the gallbladders were fixed in a $10 \%$ buffered formalin solution, and serial sections parallel to the long axis of the gallbladder were peppered and examined under a microscope. The sections were subjected to HE staining and to periodic acid-Schiff (PAS) and alcian blue double staining.

The Grimelius method was used to detect argyrophil cells and the Fontana-Masson method was chosen for the detection of argentaffin cells. Streptoavidin-biotin staining was performed in order to study gastrin, somatostatin, motilin, glucagon, CCK, lysozyme, lactoferrin, and serotonin. Non-cancerous mucosa was defined by an area not more than $1 \mathrm{~cm}$ from the cancer margin. The frequency of metaplastic cells was classified as follows: None (-), not stained at all ; mild ( + ), a small number of cells were present in part of the specimen (not more than 5-20 various intestinal metaplastic cells in a 200-magnification visual field); moderate $(++)$, metaplastic cells were clustered together in a part of the specimen (not less than 21 various intestinal metaplastic cells in a 200 -magnification visual field) ; severe $(+++)$, metaplastic cells were clustered together in various parts of specimen. 


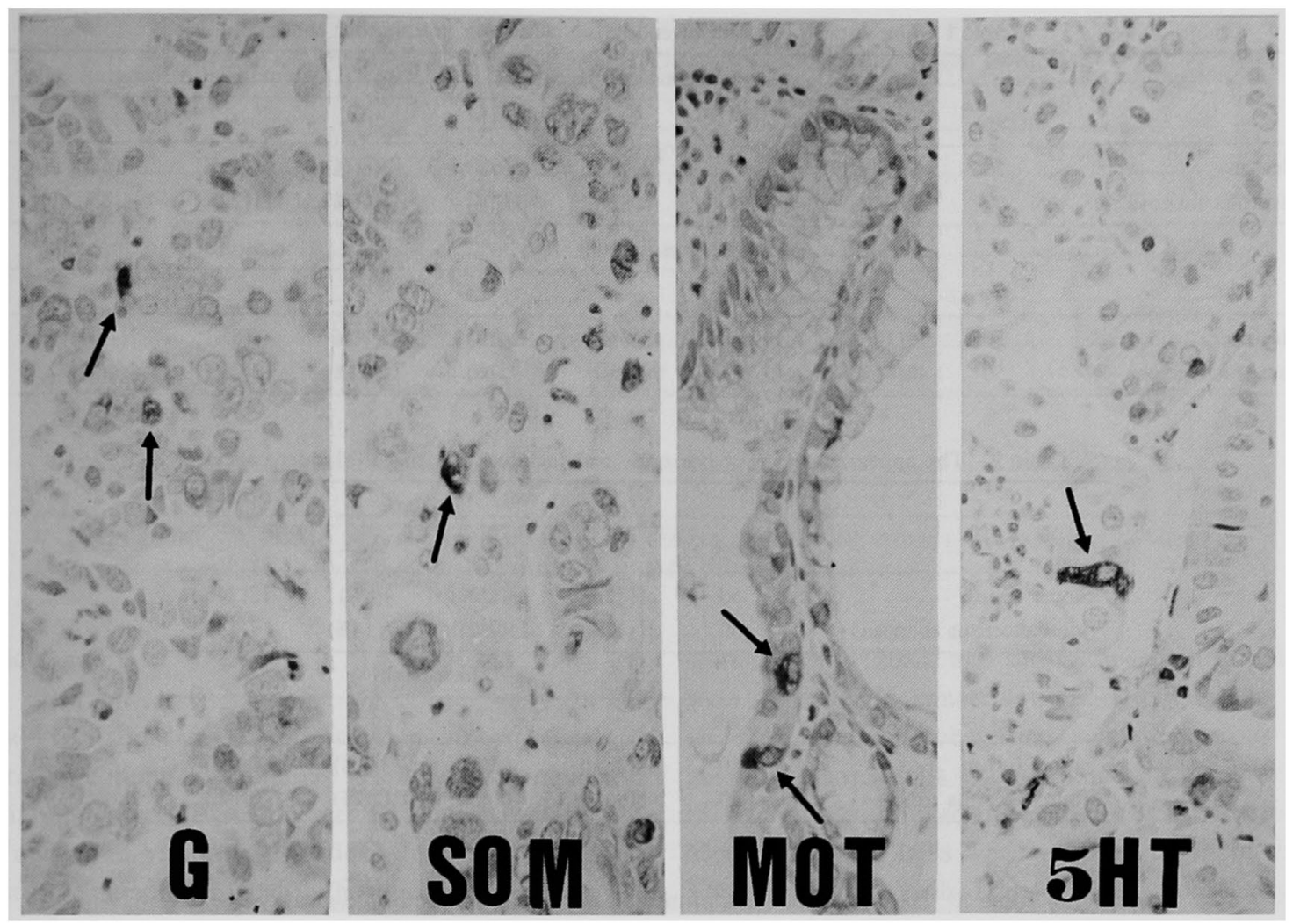

Fig. 1 Hormone-containing cells (arrows) in cancer of the gallbladder (Original manification, $\times 200$ ) G : gastrin, SOM : somatostatin, MOT : motilin, $5 \mathrm{HT}$ : serotonin

\section{Results}

Gastrin-, somatostatin- and serotonin-secreting cells were observed among the endocrine cells in immunologically-stained gallbladder cancer lesions. Motilin-secreting cells were observed only in the metaplastic cells of the non-cancerous mucosa. All immunologically-stained cells observed in cancer lesion some appear in pseudopyloric gland that considered poor differentiate morphologically and they were also observed in superficial epithelium and glandular epithelium (Fig. 1).

Among 50 cases, gastrin-secreting cells were observed in 14 cases $(28 \%)$ of cancer lesions and those were observed in 19 cases $(38 \%)$ of non-cancerous mucosa. Mildly positive reactions were observed in 11 cancerous cases (22\%) and in 14 non-cancerous cases $(28 \%)$, moderately positive reactions were observed in 3 cancerous cases $(6 \%)$ and in 4 non-cancerous cases $(8 \%)$. There were no incidences of a severely positive reaction among the cancerous lesions and only 1 case (2\%) in the non-cancerous mucosa showed a severely positive reaction. Among 50cases, somatostatin-secreting cells were observed in 10 cases (20\%) of cancer lesions and those were observed in 16cases (32\%) of non-cancerous mucosa. Mildly positive reaction was observed in 6 cancerous cases (12\%) and in 11 non-cancerous cases $(22 \%)$, moderately positive reaction was obser- ved in 3 cancerous cases $(6 \%)$ and in 4 non-cancerous cases (8\%) and severely positive reaction was observed in 1 cancerous case $(2 \%)$ and in 1 non-cancerous case (2\%). No cells from cancer lesions stained positive for the secretion of motilin, and only 2 cases (4\%) were mildly positive in non-cancerous mucosa. Among 50 cases, serotonin-secreting cells were observed in 16 cases (32\%) of cancer lesions and were observed in 19 cases (38\%) of noncancerous mucosa. Mildly positive reaction was observed in 4 cancerous cases $(8 \%)$ and in 10 non-cancerous cases (20\%), moderately positive reaction was observed in 7 cancerous cases (14\%) and in 8 non-cancerous cases $(16 \%)$ and severely positive reaction was observed in 4 cancerous cases ( $8 \%$ ) and in 1 non-cancerous case (2\%) (Table 1).

Lysozyme observed in 29 cases with cancer lesions and in 29 cases of non-cancerous mucosa as well. Mildly positive reaction was observed in 13 cancerous cases (26\%) and in 15 non-cancerous cases (30\%), moderately positive reaction was observed in 4 cancerous cases $(8 \%)$ and in 7 non-cancerous cases (14\%) and severely positive reaction was observed in 12 cancerous cases $(24 \%)$ and in 7 non-cancerous cases (14\%) (Table 2). Among 50 cases, lactofferin was appeared in 6 cases $(12 \%)$ of cancer lesion and in 4 cases $(8 \%$ ) of non-cancerous mucosa (Table 2 ).

Mildly positive reaction was observed in 5 cancerous cases (10\%) and in 4 non-cancerous cases (8\%), moderately 
Table 1 The appearance rate of endocrine cells with gallbladder cancer

\begin{tabular}{|c|c|c|c|c|c|c|c|c|c|}
\hline & \multirow[b]{2}{*}{ case } & \multicolumn{4}{|c|}{ gastrin secreting cells } & \multicolumn{4}{|c|}{ somatostatin secreting cells } \\
\hline & & - & + & ++ & +++ & - & + & ++ & +++ \\
\hline cancer lesion & 50 & $36(72 \%)$ & $11(22 \%)$ & $3(6 \%)$ & $0(0 \%)$ & $40(80 \%)$ & $6(12 \%)$ & $3(6 \%)$ & $1(2 \%)$ \\
\hline \multirow[t]{3}{*}{ non-cancerous mucosa } & 50 & $31(62 \%)$ & $14(28 \%)$ & $4(8 \%)$ & $1(2 \%)$ & $34(68 \%)$ & $11(22 \%)$ & $4(8 \%)$ & $\mathrm{I}(2 \%)$ \\
\hline & & \multicolumn{4}{|c|}{ motilin secreting cells } & \multicolumn{4}{|c|}{ serotonin secreting cells } \\
\hline & case & - & + & ++ & +++ & - & + & ++ & $++t$ \\
\hline cancer lesion & 50 & $50(100 \%)$ & $0(0 \%)$ & $0(0 \%)$ & $0(0 \%)$ & $35(70 \%)$ & $4(8 \%)$ & $7(14 \%)$ & $4(8 \%)$ \\
\hline non-cancerous mucosa & 50 & $48(96 \%)$ & $2(4 \%)$ & $0(0 \%)$ & $0(0 \%)$ & $31(62 \%)$ & $10(20 \%)$ & $8(16 \%)$ & $1(2 \%)$ \\
\hline
\end{tabular}

Table 2 The appearance rate of lysozyme and lactoferrin with gallbladder cancer

\begin{tabular}{lccccc}
\hline & & \multicolumn{4}{c}{ lysozyme } \\
\cline { 3 - 6 } & case & - & + & ++ & +++ \\
\hline cancer lesion & 50 & $21(42 \%)$ & $13(26 \%)$ & $4(8 \%)$ & $12(24 \%)$ \\
non-cancerous mucosa & 50 & $21(42 \%)$ & $15(30 \%)$ & $7(14 \%)$ & $7(14 \%)$ \\
\hline & & \multicolumn{4}{c}{ lactoferrin } \\
\cline { 3 - 6 } & case & - & + & ++ & +++ \\
\cline { 3 - 6 } & 50 & $44(88 \%)$ & $5(10 \%)$ & $1(2 \%)$ & $0(0 \%)$ \\
cancer lesion & 50 & $46(92 \%)$ & $4(8 \%)$ & $0(0 \%)$ & $0(0 \%)$ \\
non-cancerous mucosa & & & & &
\end{tabular}

Table 3 The appearance rate of metaplastic change by the histological type

\begin{tabular}{lccccccccc}
\hline & & \multicolumn{7}{c}{ non-cancerous mucosa } \\
\cline { 3 - 12 } histological type & case & GC & PGM & Paneth & Argyo & Argenta & Lz & G cell & SOM \\
\hline papillary adenoCa. & 18 & 9 & 14 & 4 & 14 & 14 & 8 & 7 & 7 \\
well diff. adenoCa. & 17 & 7 & 11 & 2 & 9 & 9 & 8 & 6 & 2 \\
mod diff. adenoCa. & 10 & 6 & 8 & 1 & 5 & 7 & 5 & 5 & 6 \\
poorly diff. adenoCa. & 5 & 1 & 4 & 0 & 2 & 2 & 0 & 1 & 1 \\
\hline \multicolumn{1}{c}{ total } & 50 & 23 & 37 & 7 & 30 & 32 & 21 & 19 & 16 \\
\hline & & & & & cancer lesion & & & \\
histological type & 18 & 10 & 9 & 2 & 11 & 15 & 13 & 7 & 3 \\
\hline papillary adenoCa. & 17 & 9 & 5 & 2 & 8 & 6 & 9 & 5 & 6 \\
well diff. adenoCa. & 10 & 7 & 4 & 1 & 3 & 3 & 7 & 2 & 1 \\
mod diff. adenoCa. & 5 & 2 & 0 & 0 & 0 & 0 & 0 & 0 & 0 \\
poorly diff. adenoCa. & 50 & 28 & 18 & 5 & 22 & 24 & 29 & 14 & 10 \\
\hline \multicolumn{1}{c}{ total } & case & GC & PGM & Paneth & Argyo & Argenta & Lz & G cell & SOM \\
\hline
\end{tabular}

GC : goblet cell, PGM : pyloric glandular metaplasia, Paneth : Paneth cell, Argyo : arbyrophill cell, Argenta : argentaffin cell, Lz: lysozyme, G cell : gastrin cell, SOM : somatostatin cell

positive reaction was observed in 1 cancerous case $(2 \%)$ in cancer lesion and not observed in non-cancerous mucosa. A severely positive reaction was not observed in either cancer lesions or in non-cancerous mucosa. The appearance rate of metaplastic changes, when examined by histological type, was relatively high in papillary adenocarcinoma. The appearance rate of various endocrine cells tended to be higher as the degree of differentiation increased. The appearance rate of argyrophilic cells, gastrin-secreting cells and somatostatin-secreting cells was significantly higher in well differentiated types compared with the poorly differentiated types. In the non-cancerous mucosa of parents with papillary cancer, metaplastic changes were high in number as well. In the non-cancerous mucosa, the appearance rate of endocrine cells and lysozyme was higher than that in the poorly differentiated adenocarcinomas. This is because the degree of differentiation increases with the moderately and well differentiated types (Table 3 ).

It is no margin of the appearance rate of each metaplastic cells between cancer lesion and non-cancerous mucosa, studied the appearance rate of metaplastic change compared with the advancement of gallbladder cancer (Table 4). 
Table 4 The appearance rate of metaplastic change by the advancement of cancer

\begin{tabular}{lrrrrrrrrrr}
\hline & & \multicolumn{7}{c}{ non-cancerous mucosa } \\
\cline { 2 - 11 } & case & GC & PGM & Paneth & Argyo & Argenta & Lz & G cell & SOM \\
\hline early cancer & 9 & 4 & 6 & 2 & 6 & 5 & 4 & 4 & 3 \\
advanced cancer & 41 & 19 & 31 & 5 & 24 & 27 & 17 & 15 & 13 \\
\hline \multicolumn{1}{c}{ total } & 50 & 23 & 37 & 7 & 30 & 32 & 21 & 19 & 16 \\
\hline & & & & & cancer lesion & & & Argy \\
\cline { 3 - 12 } & case & GC & PGM & Paneth & Argyo & Argenta & Lz & G cell & SOM \\
\hline early cancer & 9 & 5 & 4 & 1 & 5 & 4 & 6 & 3 & 2 \\
advanced cancer & 41 & 23 & 14 & 4 & 23 & 20 & 23 & 11 & 8 \\
\hline \multicolumn{1}{c}{ total } & 50 & 28 & 18 & 5 & 28 & 24 & 29 & 14 & 10 \\
\hline
\end{tabular}

GC : goblet cell, PGM : pyloric glandular metaplasia, Paneth : Paneth cell, Argyo : arbyrophill cell, Argenta : argentaffin cell, Lz : lysozyme, $\mathrm{G}$ cell : gastrin cell, SOM : somatostatin cell

\section{Discussion}

Normal gallbladder mucosa is structured uniformly and single stratum columnar epithelium is structured comparatively simple as well. It is documented that metaplastic changes appear in various gallbladder diseases ${ }^{3)}$. Of the metaplastic changes that appear in the mucosal epithelium of the gallbladder, intestinal metaplasia is attracting special attention, since it is said to play a major role in the histogenesis of gallbladder cancer ${ }^{4,5}$. There is no full report about the role of metaplastic change, lysozyme, and lactofferine in gallbladder cancer. Therefore we examined the appearance rate of endocrine cells in gallbladder cancer lesions and surrounding non-cancerous mucosa. Previous documents emphasized the existence of intestinal metaplasia; there are few reports about lysozyme, lactoferrin, and endocrine cells. In fact, intestinal epithelial cells were frequently detected in non-cancerous and cancerous tissue of the gallbladder. Therefore it is possible that these cells are included in the former lesions of gallbladder cancer.

We examined paneth cells, endocrine cells, goblet cells and lysozyme in cancer lesions and non-cancerous mucosa, considering them to be intestinal metaplasia. The appearance rate of goblet cells was in good agreement with that of the other cells. Yamamoto ${ }^{6)}$ and Nakajyou et al.") reported that the appearance of lysozyme and endocrine cells is the most useful indicator for metaplastic changes. Tsutsumi et al. ${ }^{8)}$ reported that gastrin and motilin secreting cells were found in peripheral non-cancerous mucosa in 3 out of 5 cases of gallbladder cancer. Iseki $i^{9)}$ reported that somatostatin-secreting cells were found in cancer lesions in 6 cases (16\%) and in the peripheral mucosa of 8 cases (24\%); gastrin-secreting cells were observed in peripheral mucosa of only 6 cases $(18 \%)$ among 23 cases of gallbladder cancer.

In our study, gastrin-secreting cells (28\%), somatostatin- secreting cells (20\%), and serotonin-secreting cells (30\%), were observed in cancer lesions. However, no motilinsecreting cells were observed. In non-cancerous mucosa, gastrin-secreting cells (38\%), somatostatin-secreting cells (32\%) and serotonin-secreting cells (38\%) were observed. Motilin-secreting cells were observed in $4 \%$ of the cases. These endocrine cells appeared very frequently in the cancer lesions and non-cancerous mucosa of well differentiated adenocarcinomas, while in poorly differentiated adenocarcinoma, motilin-secreting cells were observed in the non-cancerous mucosa of only 2 of the cases. These findings suggest that there is a tendency for well differentiated adenocarcinoma to develop from cases with endocrine cells. Poorly differentiated adenocarcinoma seems more likely to develop from cases without endocrine cells. In short, a strong correlation between intestinal metaplasia and endocrine cells were seen. There is a possibility of carcinogenesis in metaplastic epithelium.

In a comparison of metaplastic changes in early gallbladder cancer and advanced gallbladder cancer, no difference was found. In the non-cancerous mucosa, there was no association between metaplastic changes and the degree of differentiation, different results were obtained from cancer lesions. These results suggest that the metaplastic change is not a secondary change due to the advance of the cancer. The site of appearance of lysozyme was consistent with that of Paneth cells, with the exception of those the pseudopyloric glands. These cells have a bacteriolytic effect, which plays an important role in the local immunoresponse ${ }^{10)}$. In this study, lysozyme was observed in 29 cases (58\%) in both cancer lesions and in non-cancerous mucosa. There was a high positive reaction in 12 cancer lesions (24\%) and in 7 non-cancerous mucosa (14\%).

This finding suggests carcinogenesis with pseudopyloric glands as the background. Lactoferrin was found in only 6 cases $(12 \%)$ in cancer lesions and in 4 cases $(8 \%)$ in 
non-cancerous mucosa, suggesting that there is no association between metaplastic changes and lactoferrin. It is reported that gastrin has increased action in digestive mucosa as well as in cancer cells and somatostatin controls, which secrete various hormones ${ }^{11,12}$. In the future, we encourage studies that will survey the type and functions of many digestive endocrine cells seen in cancer lesions and peripheral non-cancerous mucosa associated with gallbladder cancer.

From these results, it is surmised that the metaplastic changes of endocrine cells and lysozyme are closely related to the development of gallbladder cancer and furthermore that such lesions are related to carcinogenesis.

\section{References}

1) Albores-Saavedra J, Nadji $M$, Henson DE. Intestinal-type adenocarcinoma of the gallbladder. A clinicopathologic and immunocytochemical study of seven cases. Am J Aurg Pathol, $10: 19-25,1986$

2) Inada $\mathbf{A}$, Konishi $\mathrm{F}$, Yamamichi $\mathrm{N}$, et al. Histogenesis of gallbladder cancer with special reference to metaplastic changes and distribution of various mucins and CEA. J Jpn Surg Soc, 90:894-906, 1989.

3) Hirai S. Clinicopathological study on metaplasia in resected gallbladder-As a background of histogenesis of gallbladder cancer. Jpn J Gastroenterol Surg, 13: 35-44, 1980.

4) Kozuka $S$, Kurashima $M$, Tsubone $M$, et al. Significance of intestinal metaplasia for the evolution of cancer in the biliary tract. Cancer, 54 : 2277-2285, 1984.

5) Hashimoto $M$. Histopathological study of the metaplasia of gallbladder carcinoma. Tando (in Japan), $9:$ 224-234, 1995.

6) Yamamoto M, Nakajyou S, Tahara E. Endocrine cells and lysozyme immunoreactivity in gallbladder. Arch Pathol Lab Med, 110: 920-927, 1986.

7) Nakajyou S, Yamamoto M, Tahara E. Histogenesis of well differentiated adenocarcinoma of the gallbladder. J Bil Panc, 8: $991-998,1987$.

8) Tsutsumi $H$, Nagura $H$, Nagamura $Y$, et al. Immunohistochemical investigation of metaplastic changes on gallbladder mucosa. Shokaki to Meneki (in Japan), 9: 78-84, 1982.

9) Iseki J. Pathological study on the histogenesis of the gallbladder carcinoma. -Intestinal metaplasia detected in gallbladder carcinoma. J Gastroenterol, 84: 1661-1667, 1987.

10) Aroni K, Kittas C, Papadimitriou CS, et al. An immunocytochemical study of distribution of lysozyme, alphal-antitripsin and alphal-antichymotrypsin in the normal and pathological gallbladder. Virchows Arch, [A] 403: 281-289, 1984.

11) Johnson LR. New aspects of the trophic action of gastrointestinal hormones. Gastroenterology, 72: 788-792, 1997.

12) Tanaka J, Takahashi T, Yamaguchi $T$, et al. The effects of gastrointestinal hormones on the growth and protein synthesis of human stomach and colon carcinomas. J Gastroenterol, 82 : 1318-1326, 1985. 